\title{
Fault Diagnosis of Ball Bearing Elements: A Generic Procedure based on Time-Frequency Analysis
}

\author{
Meng-Kun Liu, Peng-Yi Weng \\ Department of Mechanical Engineering, National Taiwan University of Science and Technology, No. 43, Sec. 4, Keelung Rd, \\ Taipei 106,Taiwan,mkliu@mail.ntust.edu.tw
}

\begin{abstract}
Motor-driven machines, such as water pumps, air compressors, and fans, are prone to fatigue failures after long operating hours, resulting in catastrophic breakdown. The failures are preceded by faults under which the machines continue to function, but with low efficiency. Most failures that occur frequently in the motor-driven machines are caused by rolling bearing faults, which could be detected by the noise and vibrations during operation. The incipient faults, however, are difficult to identify because of their low signal-to-noise ratio, vulnerability to external disturbances, and non-stationarity. The conventional Fourier spectrum is insufficient for analyzing the transient and non-stationary signals generated by these faults, and hence a novel approach based on wavelet packet decomposition and support vector machine is proposed to distinguish between various types of bearing faults. By using wavelet and statistical methods to extract the features of bearing faults based on time-frequency analysis, the proposed fault diagnosis procedure could identify ball bearing faults successfully.
\end{abstract}

Keywords: Ball bearing fault diagnosis, wavelet packet decomposition, support vector machine.

\section{INTRODUCTION}

Induction motors are used as actuators in major applications at present. A high-quality induction motor would last for decades under normal operating conditions. However, the life and efficiency of the motor could drop significantly because of various factors such as the influence of heat, vibrations, load, power variation, and mechanical wear. Deterioration in the efficiency of the motor would increase power consumption, and could even cause a catastrophic failure. An IEEE report [1] has indicated that motor failures could be categorized as electrical failure, mechanical failure, environmental contamination, etc. The report has also indicated that bearing failures are very dominant, and that they prevent the identification of other minor failures. When a ball bearing fails, it often generates abnormal vibrations and noise. Therefore, it is important to monitor the vibrations of the equipment to enable detection of bearing failures at an early stage.

The conventional vibration analysis method uses the Fourier transform. By converting a time-domain signal into its frequency counterpart, the dominant frequencies could be clearly observed. Previous studies focused on the identification of the characteristic frequency of the fault on the Fourier spectrum. However, the vibration signals were prone to contamination by the disturbances, and the accuracy of the diagnosis mainly depended on the experience of the technician. The misinterpretation of the fault symptom was mainly because of the improper representation of nonlinear and transient signals by the Fourier transform. To detect the features of such signals, time-frequency analysis methods, such as short time Fourier transform (STFT), discrete wavelet transform (DWT), and wavelet packet decomposition (WPD), were considered. The time-frequency plot generated by these methods showed a good time resolution at the high end of the frequency spectrum, and a good frequency resolution at the low end of the frequency spectrum.

In this study, it was found that the resonant frequency of the motor-driven equipment reflects the underlying timefrequency features to indicate the condition of the ball bearing. These features could be extracted by applying statistical indexes such as kurtosis, skewness, standard deviation, and root mean square to the coefficients of WPD within the bandwidth of resonant frequency. The timefrequency information of the transient impulses generated by the bearing fault could be extracted by WPD, and support vector machines (SVM) are then used as classifiers to detect the condition of the ball bearing based on these features. The proposed method does not require the machine spec in advance and complex de-noising procedure such as enveloping. It could provide precise evaluation of the ball bearing condition. 


\section{LITERATURE REVIEW}

In previous studies, Fourier spectrum has been used as the major tool to analyze ball bearing vibrations. The characteristic frequencies of the bearing faults could be derived from the bearing geometry and rotational speed. Different types of bearing faults, such as defects in the inner race, outer race, cage, and balls, could be identified based on the frequency spectrum obtained from vibration measurements [2], [3]. In several studies conducted to date, time-frequency analysis methods with machine learning algorithms have been applied to identify the bearing faults. The power spectrum densities of the fourth and the sixth level decompositions using Morlet wavelets have been proved to be good indicators of bearing faults [4]. The characteristic features of the wavelet packet energy were extracted from the WPD, and a radial basis function neural network was proposed to identify the patterns of the ball bearing faults [5]. The Daubechies 8 wavelet was demonstrated to have better diagnostic accuracy than the Symlet 8 wavelet [6]. The statistical parameters such as skewness and kurtosis were applied to the detail coefficients of DWT to distinguish between faulty and healthy bearings [7].

Time-frequency methods have often been used as the denoising tool to filter external disturbances. WPD was used as a bandpass filter to extract the critical components of the bearing fault signal, and cepstral analysis was used to separate the sidebands of the power spectrum [8]. It was proved that the cepstrum and power spectrum of the wavelet packet reconstruction could detect the outer ring fault successfully. Wavelet transform was used to de-noise the bearing vibration signal corrupted by a noisy environment [9]. The energy of WPD was then used as an index to represent the bearing health condition. It was reported that soft thresholding might result in the loss of useful information; therefore, hard thresholding was preferred for de-noising. The bearing fault signal, which was de-noised by the undecimated discrete wavelet transform (UDWT), was decomposed by empirical mode decomposition (EMD) into a set of intrinsic mode functions (IMFs) [10], [11]. The fast Fourier transform (FFT) of the specific IMF could clearly reflect the characteristic frequency of the fault. The WPD and Hilbert transform were used to extract the modulating signal of the characteristic frequency of the bearing fault [12]. In another research, sparsogram was used to identify the resonant frequency based on WPD and the enveloping method. The experimental results indicated that sparsogram was the best bearing diagnosis method compared to various other methods such as improved kurtogram, the smoothness index based WPT, and the Shannon entropy based WPT [13].

Machine learning algorithms have often been used as classifiers to distinguish faulty conditions from the normal condition. The relative spectral entropy and gravity frequency of the envelope spectrum were used as the two-dimensional vector features for the K-nearest neighbor recognition [11]. The envelope analysis based on an energy operator was reportedly better than the envelope demodulation of Hilbert transform in terms of speed and accuracy. The root mean square (RMS) values of the DWT and discrete wavelet packet transform (DWPT) were inputted to a Bayesian classifier to identify the bearing fault locations [6]. The combination of RMS and DWPT was found to achieve the best classification accuracy. The statistical indexes such as RMS, crest, and kurtosis were applied to continuous wavelet transform to generate the features for the SVM and artificial neural network (ANN). It was reported that the incipient faults were more difficult to diagnose, and that the accuracy of the SVM was higher than that of the ANN [14]. Based on WPD and five statistical features, a population based stochastic optimization technique was used to reduce the number of features and obtained high classification accuracy [15]. The signal de-noising method and Shannon entropy were applied to generate the features of each WPD level. The accuracy of the SVM classifier was $92 \%$ [16], [17]. WPD and envelope analysis were used to find the frequency band of the bearing fault. The result of distance evaluation technique, WPD coefficients, and energy were selected as the features for the SVM, and the overall classification accuracy was $95 \%$ [18]. In another study, wavelet was used as the de-noising method, and Weibull negative log-likelihood function was applied as the classifier for the time-series feature [19]. Daubechies 5 wavelet with fifth level decomposition was applied to nonextensive wavelet feature scale entropy. The locality preserving projection (LPP), which is a popular manifold learning algorithm, was then used to reduce the highdimensional features. Compared with other kernel SVMs, the SVM with Morlet wavelet kernel was reportedly the best classifier for the diagnosis of bearing faults [20].

\section{EXPERIMENTAL SETUP}

The experimental setup for the motor-driven equipment is shown in Fig.1. The setup consists of a TECO AEHF 3-phase induction motor (1 HP, low voltage squirrel cage), a TECO A510 series variable frequency drive (VFD), a Chain Tail ZKB010AA magnetic particle brake, and a Lorenz Dr-2477$P$ torque meter.

Four Wilcoxon 786A accelerometers with a sampling rate of $25,600 \mathrm{~Hz}$ were located in the vertical and the horizontal directions with respect to the bearing housing, as shown in Fig.2. There are two bearings installed in the system to support a rotating disk. The defects were only introduced to the bearing closest to the motor, and only the measurement of accelerometer 1 was taken into consideration because the horizontal vibration is most sensitive to the bearing defects from our experience. Three bearing conditions, i.e., normal condition, outer ring fault, and inner ring fault were considered in the experiment. The outer ring and inner ring faults were created by making a groove in each ring, with $0.2 \mathrm{~mm}$ depth and $0.2 \mathrm{~mm}$ width, as shown in Fig.3. The grooves were made by wire electrical discharge machining (WEDM). 


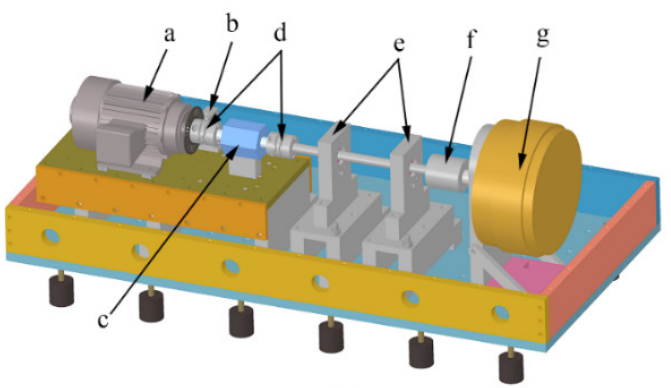

(a)

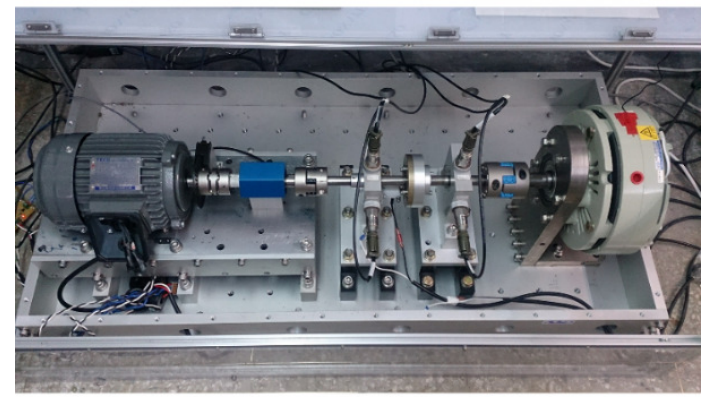

(b)

a:3-phase induction motor e:Bearing housing b:Rotary encoder f:Coupling c:Torque meter g:Magnetic particle brake d:Coupling

Fig.1. Setup for the motor-driven rotary system.

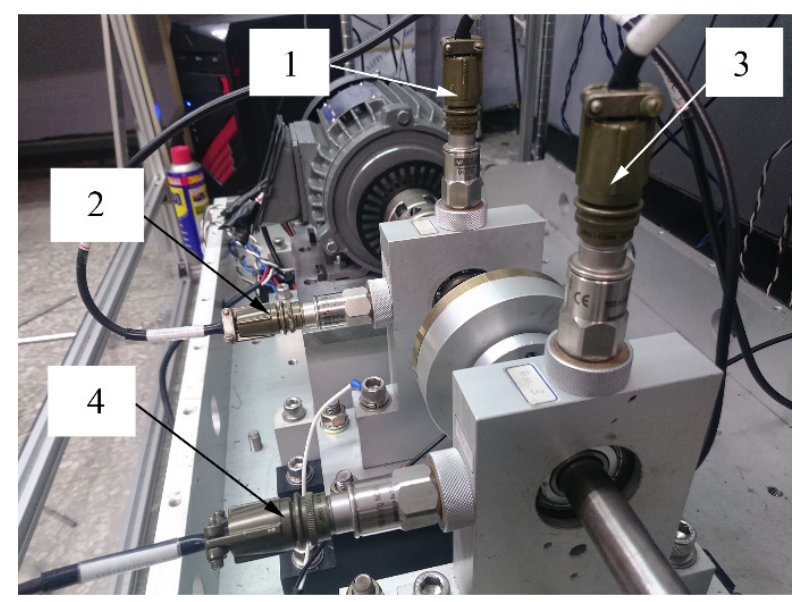

Fig.2. Installation of accelerometers.

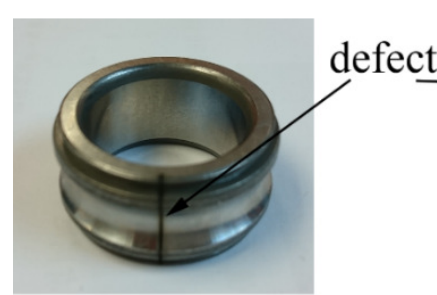

(a)

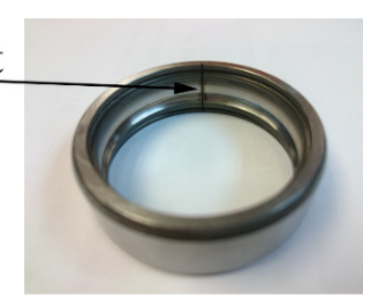

(b)
Fig.3. a) Inner ring, b) Outer ring (defect created by wire electrical discharge machining).
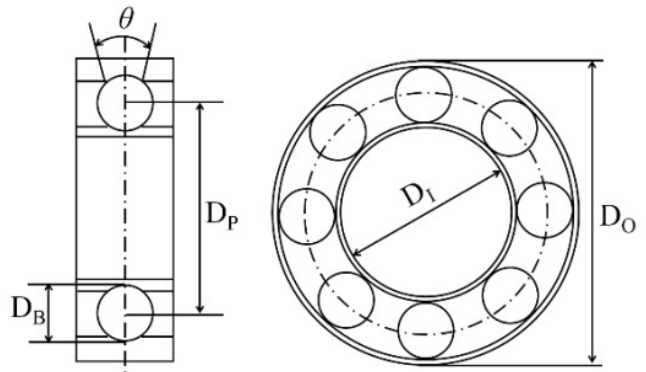

Fig.4. Dimensions of ball bearing.

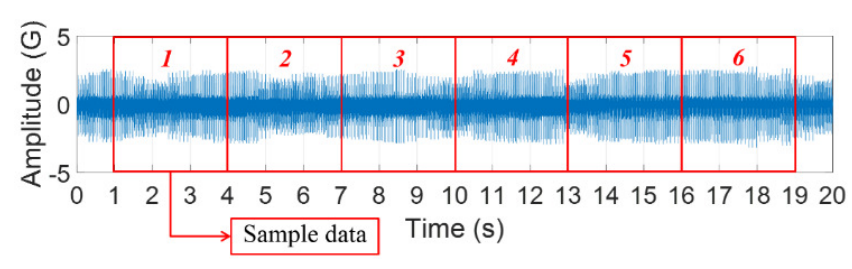

Fig.5. Processing of dataset.

Table 1. Specification of SKF 6204-T1 bearing.

\begin{tabular}{cc}
\hline $\begin{array}{c}\text { Geometry } \\
\text { parameter }\end{array}$ & Size \\
\hline Outer diameter $\left(D_{O}\right)$ & $47 \mathrm{~mm}$ \\
Inner diameter $\left(D_{I}\right)$ & $20 \mathrm{~mm}$ \\
Pitch diameter $\left(D_{P}\right)$ & $33.5 \mathrm{~mm}$ \\
Ball diameter $\left(D_{B}\right)$ & $8 \mathrm{~mm}$ \\
Number of balls $\left(N_{B}\right)$ & 8 \\
Contact angle $(\theta)$ & $0^{\circ}$ \\
\hline
\end{tabular}

The speed of the motor could be adjusted by tuning the output frequency of the VFD. The VFD frequencies in the experiment were set to $45 \mathrm{~Hz}$ and $60 \mathrm{~Hz}$, equivalent to motor speeds of $1350 \mathrm{rpm}$ and $1800 \mathrm{rpm}$, respectively. Because the characteristic frequencies of motor faults are usually defined in the unit of $\mathrm{Hz}$, the unit of rpm is converted to the unit of $\mathrm{Hz}$ in this study. The recorded data included three-phase voltage and current signals, and the signals from the four accelerometers, rotary encoder, and torque meter. Once the motor speed reached steady state, the data were recorded for a span of $20 \mathrm{~s}$ in each experiment. The experiments were conducted at two speeds: $22.5 \mathrm{~Hz}$ and $30 \mathrm{~Hz}$. At each speed, the tests covered three different bearing conditions, i.e., healthy condition, with inner ring defect, and with outer ring defect. Each experiment was repeated three times. The parameters of the ball bearing are shown in Fig.4., and the dimensions are presented in Table 1. Resistance to rotation and eccentricity were the other control variables in the experiment. Three different torques, i.e., $0.3 \mathrm{Nm}, 1.5 \mathrm{Nm}$, and $3 \mathrm{Nm}$, and three eccentricity conditions, i.e., normal, angular eccentricity, and parallel eccentricity, were applied. The eccentricities were introduced between the motor and the 
closest bearing housing by adjusting their relative positions, and the amounts of eccentricities were measured by using a laser alignment system (FixturLaser P-0272-GB). In the experiment, the parallel and angular eccentricities were intentionally made to $0.3 \mathrm{~mm}$ and $0.25 / 100(0.25 \mathrm{~mm}$ deviation per $100 \mathrm{~mm}$ length), respectively, which greatly exceed the normal values. However, the influence of the eccentricities to the vibration measurement is not obvious, so we did not regard the eccentricities as effective failure modes. From the bearing specifications presented in Table 1., the characteristic frequencies of the bearing faults were calculated using (1)-(4); the frequencies are presented in Table 2.

$$
F_{C F}=\frac{1}{2} F_{R}\left(1-\frac{D_{B} \cos (\theta)}{D_{P}}\right)
$$

$$
\begin{gathered}
F_{\text {ORF }}=\frac{N_{B}}{2} F_{R}\left(1-\frac{D_{B} \cos (\theta)}{D_{P}}\right) \\
F_{I R F}=\frac{N_{B}}{2} F_{R}\left(1+\frac{D_{B} \cos (\theta)}{D_{P}}\right) \\
F_{B F}=\frac{D_{P}}{2 D_{B}} F_{R}\left(1-\frac{D_{B}^{2} \cos ^{2}(\theta)}{D_{P}^{2}}\right)
\end{gathered}
$$

To increase the number of data sets, the time span of $20 \mathrm{~s}$ for the data of each experiment was divided into six segments, as shown in Fig.5. Thus, the time span of each segment was $3 \mathrm{~s}$. As each experiment was repeated three times, there were

\begin{tabular}{|c|c|c|c|c|c|c|}
\hline No. & VFD (Hz) & $\begin{array}{c}\text { Rotation } \\
\text { speed }(\mathbf{H z})\end{array}$ & $\begin{array}{l}\text { Resistance } \\
\text { A }(0.3 \mathrm{Nm}) \\
\text { B }(1.5 \mathrm{Nm}) \\
C(3.0 \mathrm{Nm}) \\
\end{array}$ & $\begin{array}{l}\text { Bearing failure } \\
\text { mode }\end{array}$ & $\begin{array}{c}\text { Bearing } \\
\text { characteristic } \\
\text { frequency }(\mathrm{Hz})\end{array}$ & Eccentricity \\
\hline 1 & 45 & 22.5 & $\mathrm{~A}$ & Inner ring fault & 111.2 & Angular eccentricity \\
\hline 2 & 45 & 22.5 & B & Outer ring fault & 68.7 & Normal \\
\hline 3 & 45 & 22.5 & $\mathrm{C}$ & Normal & $\mathrm{N} / \mathrm{A}$ & Parallel eccentricity \\
\hline 4 & 60 & 30 & $\mathrm{~A}$ & Outer ring fault & 91.7 & Parallel eccentricity \\
\hline 5 & 60 & 30 & B & Normal & $\mathrm{N} / \mathrm{A}$ & Angular eccentricity \\
\hline 6 & 60 & 30 & $\mathrm{C}$ & Inner ring fault & 148.3 & Normal \\
\hline
\end{tabular}
18 data sets for each condition. As a result, the total number of data sets in Table 2. is 54 .

Table 2. Parameters for the experiment.

Frequency sweeping was conducted before the start of the experiment. This was an important step in the investigation of the resonant frequencies of the motor-driven equipment. The waterfall plots for the normal condition, inner ring fault, and outer ring fault are shown in Fig.6. Two types of resonance phenomena were observed: static and dynamic. The static resonant frequency remained unchanged when the rotation speed was varied. On the contrary, the dynamic resonant frequency was proportional to the rotation speed. The waterfall plot for the normal condition is shown in Fig.6.a); it shows five groups of static resonant frequencies, $0-1500 \mathrm{~Hz}, 2500-3500 \mathrm{~Hz}, 3500-4500 \mathrm{~Hz}, 7000-8000 \mathrm{~Hz}$, and $10000-12000 \mathrm{~Hz}$. In addition, a few dynamic resonant frequencies could be observed between 0 to $3000 \mathrm{~Hz}$. The waterfall plots for the inner and outer ring fault conditions are shown in Fig.6.b) and Fig.6.c), respectively. It can be seen from the figures that multiple frequencies are triggered, especially around the static resonant frequency of $3000 \mathrm{~Hz}$, because of the impact between the ball and the defect on the ring. However, the difference between the Fourier spectra of the inner and outer ring faults was not significant.

\section{ANALYSIS METHOD}

The conventional Fourier analysis adopts sinusoidal basis functions to convert the time-domain signal into its frequency counterpart. These sinusoidal basis functions, however, are global functions, and hence the methods based on Fourier spectrum cannot reflect the genuine characteristics of a transient, non-stationary signal. On the other hand, continuous wavelet transform can be used in time-frequency analysis by dilating and translating the local basis, called mother wavelet. It has a good frequency resolution at the low end of the frequency spectrum and a good time resolution at the high end of the frequency spectrum. The discrete form of the continuous wavelet transform can be represented as

$$
\operatorname{DWT}(j, k)=\frac{1}{\sqrt{2^{j}}} \sum_{n} x(n) \Psi\left(\frac{n-k 2^{j}}{2^{j}}\right)
$$

where $\mathrm{j}, \mathrm{k}, \mathrm{n} \in \mathrm{Z}$. The DWT could be realized by feeding the time-domain signal into a series of high pass and low pass filter pairs. The approximation coefficients and detail coefficients are generated when the time-domain signal is convoluted with low pass filter and high pass filter, respectively. As the DWT applies high pass and low pass filter pairs only to the approximation coefficients, it has limited bandwidth especially at the high frequency spectrum. However, the WPD shown in Fig.7. applies the filter pairs to both approximation and detail coefficients. Hence it has better frequency resolution at the high frequency spectrum than the DWT. The WPD coefficients at each level are defined by the following equations. 


$$
\begin{gathered}
W_{j+1}^{2 K}=W_{j}^{K}(n) * h(-2 n) \\
W_{j+1}^{2 K+1}=W_{j}^{K}(n) * g(-2 n)
\end{gathered}
$$

where $W_{j+1}^{2 K}$ denotes the $j$-th decomposed level of the wavelet packet coefficients in the frequency band of $2 k(0<k<$ $\left.2^{j}-1\right)$. The terms $h(-2 n)$ and $g(-2 n)$ are the low-pass and high-pass filters, respectively, based on the selected mother wavelet function.

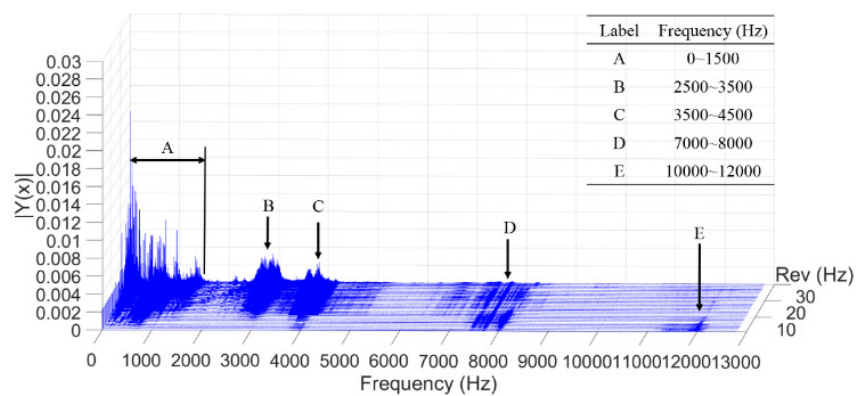

a)

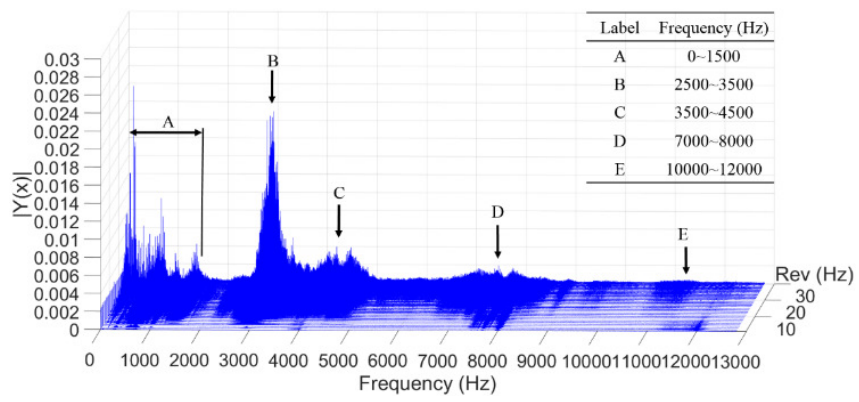

b)

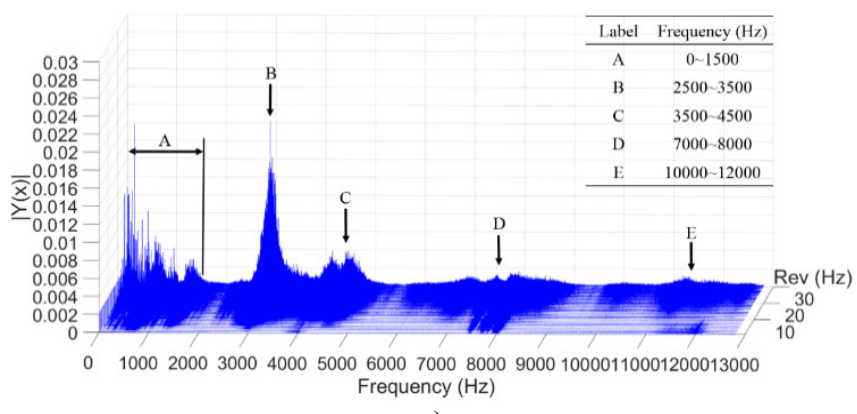

c)

Fig.6. Waterfall plot of resonant frequencies under a) normal condition, b) inner ring fault, c) outer ring fault.

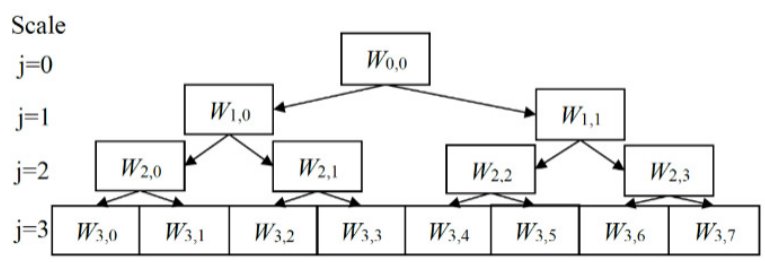

Fig.7. Three-layer wavelet packet decomposition (WPD).

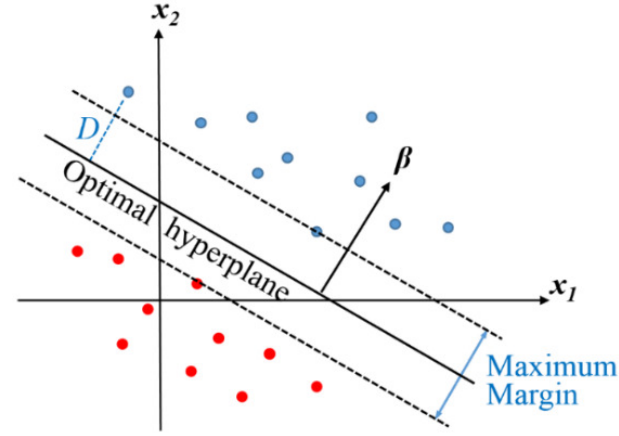

Fig.8. Hyperplane of support vector machine.

Statistical indexes such as kurtosis, skewness, standard deviation, root mean square, and maximum value were extracted from the wavelet packet coefficients. These statistical indexes are defined by the following equations.

$$
\begin{gathered}
\text { Kurtosis }=\frac{\frac{1}{n} \sum_{i=1}^{n}\left(x_{i}-\bar{x}\right)^{4}}{\left(\frac{1}{n} \sum_{i=1}^{n}\left(x_{i}-\bar{x}\right)^{2}\right)^{2}} \\
\text { Skewness }=\frac{\frac{1}{n} \sum_{i=1}^{n}\left(x_{i}-\bar{x}\right)^{3}}{\left(\frac{1}{n} \sum_{i=1}^{n}\left(x_{i}-\bar{x}\right)^{2}\right)^{\frac{3}{2}}} \\
\text { S.D. }=\sqrt{\frac{1}{n} \sum_{i=1}^{n}\left(x_{i}-\bar{x}\right)^{2}} \\
\text { RMS }=\sqrt{\frac{1}{n} \sum_{i=1}^{n} x_{i}^{2}} \\
\text { Max. }=\max (x)
\end{gathered}
$$

where $\bar{x}$ is the average of the data and $n$ is the sample size. SVM was used as the classifier to detect the ball bearing condition. In this algorithm, the data were plotted in a multidimensional space with the coordinates defined by the values of their features. The operation of the SVM algorithm relies on finding the hyperplane that gives the largest minimum distance to the training examples. The minimum distance is called margin in SVM terminology. Therefore, the optimal separation hyperplane is the one that maximizes the margin of the training data, as shown in Fig.8. The equation for the hyperplane can be defined as

$$
\mathbf{f}(x)=\beta^{\mathrm{T}} x+\beta_{0}
$$

where $\beta$ is the weight vector and $\beta_{0}$ is the bias. The optimal hyperplane can be represented in an infinite number of 
different ways by scaling $\beta$ and $\beta_{0}$. As a matter of convention, among all the possible representations of the hyperplane, the one chosen is

$$
\left|\beta^{\mathrm{T}} x+\beta_{0}\right|=1
$$

where $x$ refers to the training examples closest to the hyperplane. In general, the training examples that are closest to the hyperplane are called support vectors. The hyperplane that meets the condition mentioned above is known as the canonical hyperplane. The distance $\mathrm{D}$ between a point $\mathrm{x}$ and the hyperplane $\left(\beta, \beta_{0}\right)$ is calculated using the following equation.

$$
D=\frac{\left|\beta^{\mathrm{T}} x+\beta_{0}\right|}{\|\beta\|}
$$

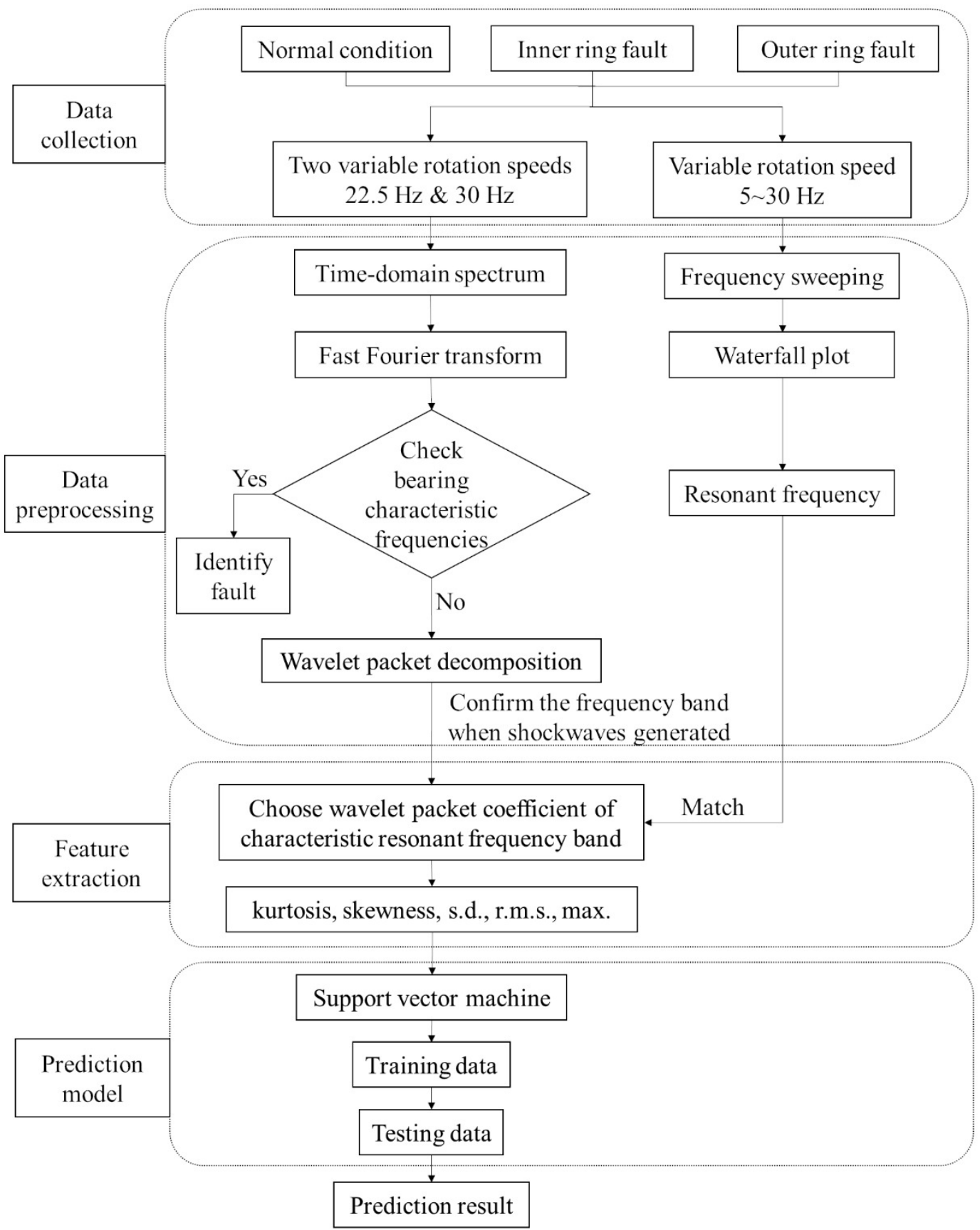

Fig.9. Flowchart of the diagnosis scheme. 
In particular, for the canonical hyperplane, the numerator is equal to one and the distance to the support vectors is given by

$$
\text { distance }_{\text {support vector }}=\frac{\left|\beta^{\mathrm{T}} x+\beta_{0}\right|}{\|\beta\|}
$$

The maximum margin introduced in Fig.8., denoted here as $\mathrm{M}$, is twice the distance to the closest training examples

$$
M=\frac{2}{\|\beta\|}
$$

Finally, the problem of maximizing $M$ is equivalent to the problem of minimizing a function $\mathrm{L}(\beta)$ subject to some constraints. The requirements for the hyperplane are decided based on the constraints to classify all the training examples $x_{i}$ correctly.

$$
\min _{\beta, \beta_{0}} \mathrm{~L}(\beta)=\frac{1}{2}\|\beta\|^{2} \text { subject to } y_{i}\left(\beta^{\mathrm{T}} x+\beta_{0}\right) \geq 1 \forall i
$$

where $y_{i}$ represents the labels of the training examples.

The analysis procedure proposed in this research consists of four stages: data collection, data pre-processing, feature extraction, and model prediction. The details are shown in Fig.9. At the data collection stage, both frequency sweeping and the experiments as per the details presented in Table 2. were conducted. Then, waterfall plots were developed at the data pre-processing stage to confirm the resonant frequency, while Fourier spectrum was used to detect the characteristic frequencies of the bearing faults. WPD was then applied to analyze the vibration signals. At the feature extraction stage, five statistical indexes were applied to the coefficients of WPD that are related to the main resonant bandwidth. Finally, the SVM was constructed at the model prediction stage.

\section{EXPERIMENTAL RESULTS AND DISCUSSION}

The time-domain response, frequency spectrum, and the WPD of the vibration signals acquired from accelerometer 1 in each condition are shown in Fig.10., Fig.11., and Fig.13. The sixth level decomposition with $\mathrm{db} 20$ as the mother wavelet was chosen empirically for the WPD, and the rotating speed was set to fs $=22.5 \mathrm{~Hz}$. The frequency spectrum of the vibrations in the case of a healthy bearing, shown in Fig.10.b), indicates that there are two groups of frequencies around 0-1000 Hz and $4000 \mathrm{~Hz}$. The plot of WPD shown in Fig.10.c) also indicates the same result. The frequencies around $3000 \mathrm{~Hz}$ were not significant.

When the bearing with the inner ring defect was tested, periodic shockwaves caused by the collision of the balls and the defect were detected on the time-domain response, as shown in Fig.11.a). The periods (and frequencies) of the shockwaves were $0.0096 \mathrm{~s}(104 \mathrm{~Hz})$ and $0.046 \mathrm{~s}(21.5 \mathrm{~Hz})$. These frequencies matched the ball pass frequencies of the inner ring fault (BPFI) and the rotation speed (fs). Because of the resisting torque applied in the experimental setup, the actual rotation speed was slower than the theoretical value. The long period of the shockwave was because of the clearance between the balls and the inner ring, which makes the collision between them occur once per cycle, as shown in Fig.12.a).

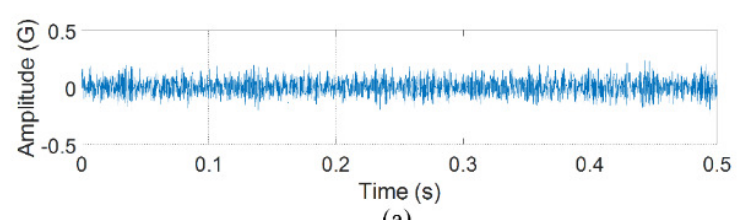

(a)
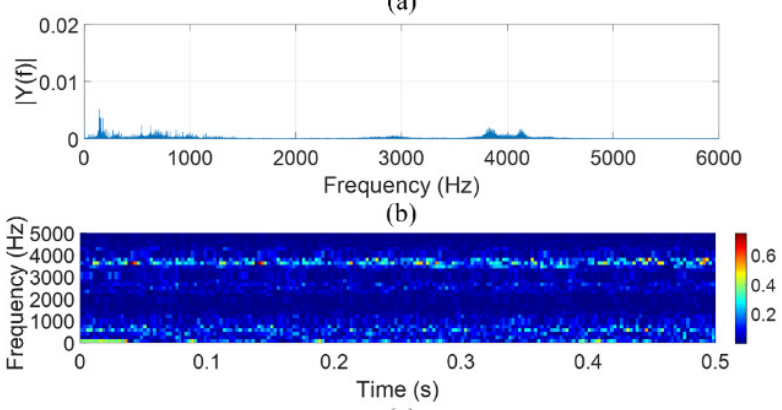

(c)

Fig.10. a) Time-domain response, b) FFT, c) WPD of healthy bearing with $f_{S}=22.5 \mathrm{~Hz}$.

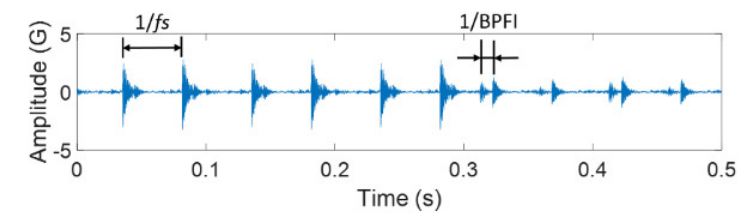

(a)
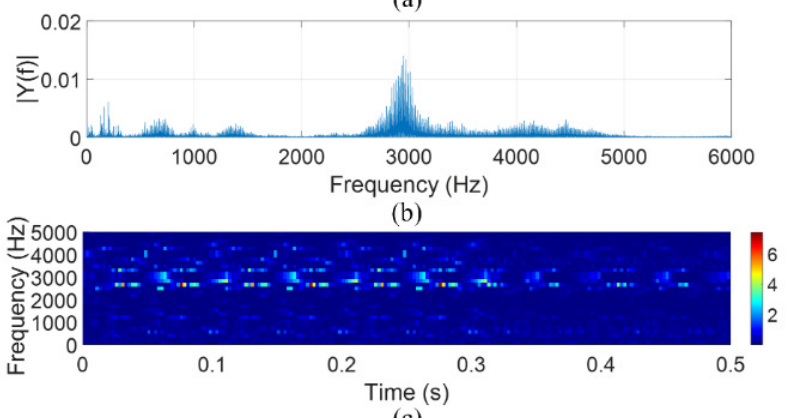

(c)

Fig.11. a) Time-domain response, b) FFT, c) WPD of inner ring fault with $f_{S}=22$. Hz.

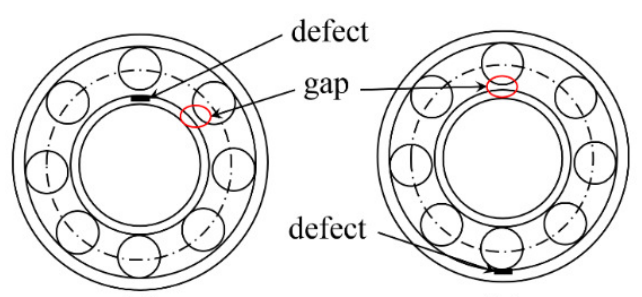

(a)

(b)

Fig.12. Relationship between defect and gap: a) Inner ring fault, b) Outer ring fault. 
Both the Fourier spectrum and WPD plot, shown in Fig.11.b) and Fig.11.c), respectively, indicate that the resonant frequencies were triggered around $3000 \mathrm{~Hz}$. Likewise, when the bearing with the outer ring defect was tested, periodic shockwaves caused by the collision of the balls and defect were detected, as shown in Fig.13.a). In the case of the outer ring defect, there was no clearance between the ball and the defect, as shown in Fig.12.b), because the gravity forced them to remain in close contact. There was only one period of $0.01461 \mathrm{~s}(68.5 \mathrm{~Hz})$ for the shockwaves, equivalent to the ball pass frequency of the outer ring fault (BPFO). Both the Fourier spectrum and WPD plot were similar to those in Fig.11., and the resonant frequency of $3000 \mathrm{~Hz}$ could be observed in both Fig.13.b) and Fig.13.c).

From Fig.13., it can be concluded that the characteristics of the transient and non-stationary shockwaves could not be identified using the Fourier spectrum, but their pattern could be reflected by the WPD. The resonant frequencies were observed around $3000 \mathrm{~Hz}$ in the spectrum of the $50^{\text {th }}$ node in the sixth level wavelet packet decomposition. Hence, the statistical indexes such as kurtosis, skewness, standard deviation, root mean square value, and maximum value were applied to the WPD coefficients for the $50^{\text {th }}$ node for generating the features to be inputted to the SVM.

As mentioned previously, the data were recorded with a time span of $20 \mathrm{~s}$, and were divided into six segments for each experiment. Each experiment was repeated three times. The statistical indexes for the three rounds of experiments with $f_{s}=22.5 \mathrm{~Hz}$ are shown in Fig.14. The normal and faulty conditions could be clearly distinguished by most of the indexes, except kurtosis and skewness, as shown in Fig.14.a) and Fig.14.b). It was not possible to distinguish clearly between the inner ring fault and the outer ring fault using these two indexes. In general, the values of the inner ring fault indexes, however, were larger than those of the outer ring fault indexes. Data from any two rounds were used as the training data sets for the SVM, and the data from the remaining round were used as the testing data set; thus, there would be three sets of accuracies after interchanging the rounds.

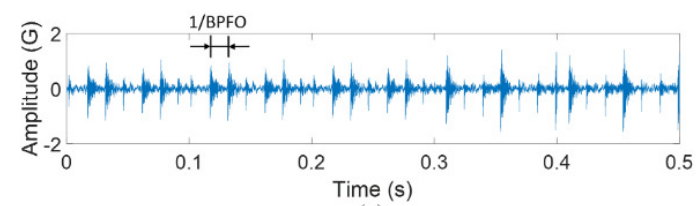

(a)

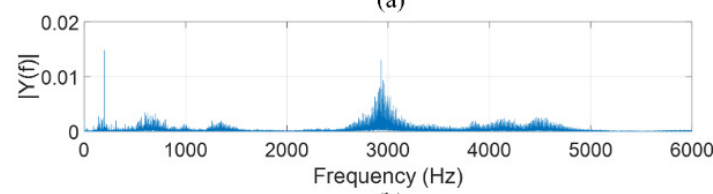

(b)

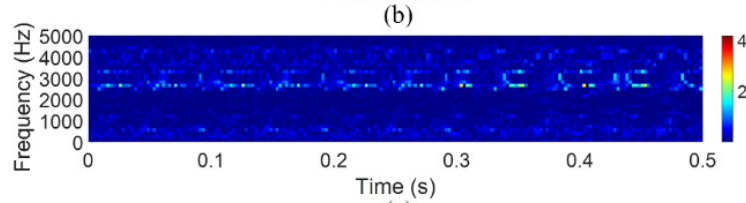

(c)

Fig.13. a) Time-domain response, b) FFT, c) WPD of outer ring fault with $f_{s}=22.5 \mathrm{~Hz}$.

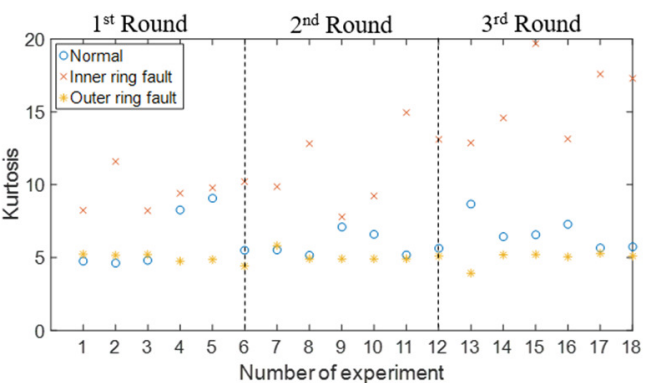

a)

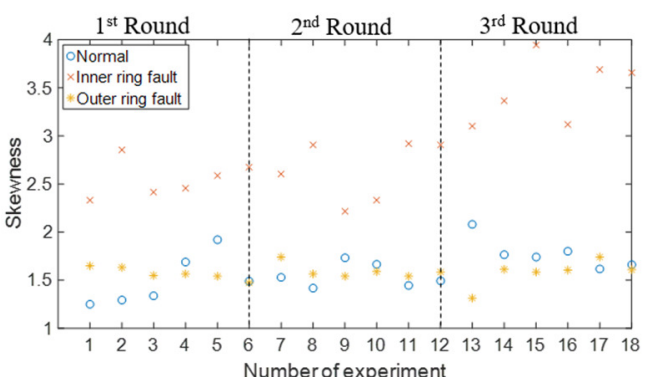

b)

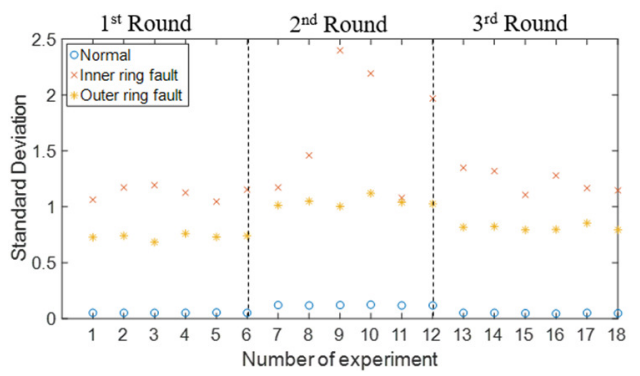

c)

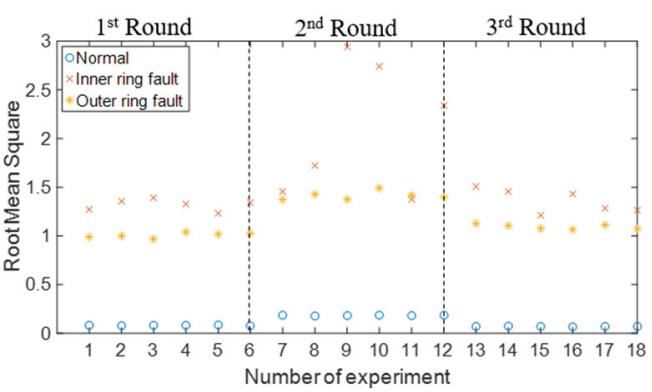

d)

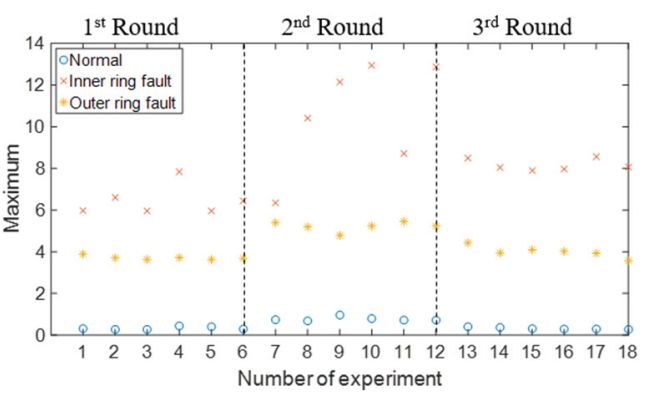

e)

Fig. 14. a) Kurtosis , b) skewness, c) standard deviation, d)root mean square, and e)maximum value for each round $\left(f_{S}=22.5 \mathrm{~Hz}\right)$. 
An overall accuracy of $97.9 \%$ was obtained from the calculations, as presented in Table 3. Similarly, the features in the three rounds corresponding to $f_{s}=30 \mathrm{~Hz}$ also obtain an overall accuracy of $98.3 \%$, as presented in Table 4 .

Table 3. Classification accuracy of SVM $(f s=22.5 \mathrm{~Hz})$.

\begin{tabular}{ccc}
\hline Training data & Testing data & Accuracy \\
\hline $\begin{array}{c}\text { 1st round } \\
\text { 2nd round }\end{array}$ & 3rd round & $98.2 \%$ \\
\hline $\begin{array}{c}\text { 1st round } \\
\text { 3rd round }\end{array}$ & 2nd round & $99.0 \%$ \\
\hline $\begin{array}{l}\text { 2nd round } \\
\text { 3rd round }\end{array}$ & 1st round & $96.5 \%$ \\
\hline \multicolumn{3}{c}{ Overall accuracy $=\mathbf{9 7 . 9 \%}$} \\
\hline
\end{tabular}

Table 4. Classification accuracy of SVM $\left(f_{S}=30 \mathrm{~Hz}\right)$.

\begin{tabular}{ccc}
\hline Training data & Testing data & Accuracy \\
\hline $\begin{array}{l}\text { 1st round } \\
\text { 2nd round }\end{array}$ & 3rd round & $98.5 \%$ \\
\hline $\begin{array}{l}\text { 1st round } \\
\text { 3rd round }\end{array}$ & 2nd round & $99.2 \%$ \\
\hline $\begin{array}{l}\text { 2nd round } \\
\text { 3rd round }\end{array}$ & 1st round & $97.3 \%$ \\
\hline \multicolumn{2}{c}{ Overall Accuracy $=\mathbf{9 8 . 3} \%$}
\end{tabular}

\section{CONCLUSIONS}

A generic fault diagnosis procedure was proposed in this research for detecting the ball bearing faults in motor-driven equipment. The experiments were conducted on bearings in normal condition, with inner ring defect, and with outer ring defect, under various resistance levels and rotational speeds. Before the experiments were conducted, waterfall plots were developed to identify the static and dynamic resonances. Periodic shockwaves were observed in the time domain when the bearings with inner ring or outer ring defect were tested. Although the characteristic frequency of the fault could be predicted based on the bearing specification, the frequency was not significant on the Fourier spectrum, because the conventional Fourier spectrum is insufficient for analyzing the transient and non-stationary shockwaves. Hence, wavelet packet decomposition was applied, and it was found that the distinct time-frequency features could be extracted from the coefficients at the resonant frequency. Five statistical indexes, i.e., kurtosis, skewness, standard deviation, root mean square, and maximum value, were applied to the coefficients of the wavelet packet decomposition for generating the inputs to the support vector machine to classify the failure modes. Because only the information of resonant frequencies is required to conduct the analysis, the proposed method could be applied to multiple types of induction motors with different rotation speeds. It was proved to be effective and accurate for identifying the ball bearing condition. The same procedure that adopts time-frequency information to diagnose the abnormality could be applied to other rotary machines as well.

\section{ACKNOWLEDGMENT}

This work was financially supported by the 'Center for Cyber-physical System Innovation' from The Featured Area Research Center Program within the framework of the Higher Education Sprout Project by the Ministry of Education (MOE) in Taiwan. Part of the funding also came from the Ministry of Science and Technology (MOST) in Taiwan under Grant no. MOST 106-2221-E-011-086.

\section{REFERENCES}

[1] Bell, R., McWilliams, D., O'Donnell, P., Singh, C., Wells, S. (1985). Report of large motor reliability survey of industrial and commercial installations, Part I. IEEE Transactions on Industry Applications, IA-21 (4), 853-864.

[2] Kumar, A., Mishra, A. (2014). Bearing fault diagnosis based on vibration signature analysis using discrete wavelet transform. IJERT - International Journal of Engineering Research \& Technology, 3 (8), 1258-61.

[3] Attoui, I., Boutasseta, N., Fergani, N., Oudjani, B., Deliou, A. (2015). Vibration-based bearing fault diagnosis by an integrated DWT-FFT approach and an adaptive neuro-fuzzy inference system. In 2015 3rd International Conference on Control, Engineering \& Information Technology (CEIT). IEEE, 1-6.

[4] Jeevanand, S., Mathew, A.T. (2008). Condition monitoring of induction motors using wavelet based analysis of vibration signals. In 2008 Second International Conference on Future Generation Communication and Networking Symposia. IEEE, Vol. 3, 75-80.

[5] Fang, S., Zijie, W. (2007). Rolling bearing fault diagnosis based on wavelet packet and RBF neural network. In 2007 Chinese Control Conference. IEEE, 451-455.

[6] Chebil, J., Noel, G., Mesbah, M., Deriche, M. (2009). Wavelet decomposition for the detection and diagnosis of faults in rolling element bearings. Jordan Journal of Mechanical and Industrial Engineering, 3 (4), 260-267.

[7] Patel, R.K., Giri, B.R. (2015). Application of DWT and PDD for bearing fault diagnosis using vibration signal. Journal of Electrical Engineering, 15 (4), 139144.

[8] Ma, J., Wu, J., Wang, X., Fan, Y., Leng, T. (2013). A fault detection method of rolling bearing based on wavelet packet-cepstrum. Research Journal of Applied Sciences, Engineering and Technology, 5 (12), 34023406.

[9] Kulkarni, P.G., Sahasrabudhe, A.D. (2013). Application of wavelet transform for fault diagnosis of rolling element bearings. International Journal of Scientific \& Technology Research, 2 (4), 138-148.

[10] Wei, Z., Gao, J., Zhong, X., Jiang, Z., Ma, B. (2011). Incipient fault diagnosis of rolling element bearing based on wavelet packet transform and energy operator. WSEAS Transactions on Systems, 10 (3), 8190. 
[11] Raj, A.S., Murali, N. (2013). Morlet wavelet UDWT denoising and EMD based bearing fault diagnosis. Electronics, 17 (1), 1-8.

[12] Cung, L.E., Hien, B.M., Son, N.T. (2016). Gear and bearing fault detection using wavelet packet and Hilbert method via acoustic signals. ASEAN Engineering Journal Part A, 6 (2), 1-9.

[13] Tse, P.W., Wang, D. (2013). The automatic selection of an optimal wavelet filter and its enhancement by the new sparsogram for bearing fault detection: Part 2 of the two related manuscripts that have a joint title as "Two automatic vibration-based fault diagnostic methods using the novel sparsity measurement-Parts 1 and 2". Mechanical Systems and Signal Processing, 40 (2), 520-544.

[14] Konar, P., Chattopadhyay, P. (2011). Bearing fault detection of induction motor using wavelet and Support Vector Machines (SVMs). Applied Soft Computing, 11 (6), 4203-4211.

[15] Rajeswari, C., Sathiyabhama, B., Devendiran, S., Manivannan, K. (2014). Bearing fault diagnosis using wavelet packet transform, hybrid PSO and support vector machine. Procedia Engineering, 97, 1772-1783.

[16] Xu, Y.J., Xiu, S.D. (2011). A new and effective method of bearing fault diagnosis using wavelet packet transform combined with support vector machine. Journal of Computers, 6 (11), 2502-2509.
[17] Wenxing, M., Meng, L. (2008, July). Fault pattern recognition of rolling bearings based on wavelet packet and support vector machine. In 2008 27th Chinese Control Conference. IEEE, 65-68.

[18] Hu, Q., He, Z., Zhang, Z., Zi, Y. (2007). Fault diagnosis of rotating machinery based on improved wavelet package transform and SVMs ensemble. Mechanical Systems and Signal Processing, 21 (2), 688-705.

[19] Abbasion, S., Rafsanjani, A., Farshidianfar, A., Irani, N. (2007). Rolling element bearings multi-fault classification based on the wavelet denoising and support vector machine. Mechanical Systems and Signal Processing, 21 (7), 2933-2945.

[20] Dong, S., Tang, B., Chen, R. (2013). Bearing running state recognition based on non-extensive wavelet feature scale entropy and support vector machine. Measurement, 46 (10), 4189-4199

Received February 6, 2019

Accepted July 30, 2019 